\title{
Review
}

\section{External Negative Pressure Dressing System(ENDPS) $V S$. Traditional Wound Dressing for Cesarean Section Incision in Obese Women: A Randomized Controlled Trial}

\author{
Dalia MM Muhammad*, Mohammed SEl Sokkary, Marwan O Elkady \\ Department of Obstetrics \& Gynaecology, Ain Shams University, Cairo, Egypt
}

*Correspondence to: Dalia Magdy Mokhtar Muhammad, Department of Obstetrics \& Gynaecology, Ain Shams University, Cairo, Egypt; E-mail: courageous_life@ yahoo.com

Received: Dec 15 ${ }^{\text {th }}, 2020$; Accepted: Feb $5^{\text {th }}, 2021$; Published: Feb 17 ${ }^{\text {th }}, 2021$

Citation: Muhammad DMM, SEl-Sokkary M, Elkady MO. External negative pressure dressing system(ENDPS) VS. traditional wound dressing for cesarean section incision in obese women: A randomized controlled trial. Biom Case Rep Open A Open J. 2021; 2(1): 58-62. doi: 10.33169/biomcase. BACROAOJ-2-116

\begin{abstract}
What is already known on this subject? AND What does this study add? Obesity is associated with increased cesarean section delivery rates and surgical site wound complication with associated increased post-operative morbidity, post-operative pain and length of hospital stay. What does this study add? Negative pressure wound therapy (NPWT) technology could be used as a prophylactic measure to reduce surgical site wound complications in obese women undergoing cesarean section by immediate postoperative application in clean-contaminated, closed surgical incisions.
\end{abstract}

Keywords: Obesity, Epithelialization, Fibroplasia, Cesarean section, Cytokines and elongate.

\section{INTRODUCTION}

Obesity, defined as body mass index (BMI, calculated as weight $(\mathrm{kg}) /$ [height (m)] 2) of $30 \mathrm{~kg} / \mathrm{m} 2$ or greater, is a common medical comorbidity of pregnancy affecting one third of reproductive-aged women. Maternal obesity is also a well-recognized risk factor for dysfunctional labor and cesarean delivery with a cesarean section rate of $33 \%$ in obese women with BMI of 30 or greater and $43 \%$ in women with BMIs of 40 or greater.

Obesity is an independent risk factor for post-operative surgical site wound complications. The risk of post-cesarean surgical site wound complications has been shown to double for every 5 unit increase in body mass index (BMI) above $30 \mathrm{~kg} / \mathrm{m}$, occurring in about $10 \%$ of obese women undergoing caesarean section despite prophylactic strategies (e.g. antibiotics). ${ }^{2}$

Wound healing is a sequence of physiologic events that include inflammation, epithelialization, fibroplasia, and maturation. Fail- ure of wound healing at the surgical site can lead to seroma, hematoma, wound dehiscence, incisional hernia and surgical site infection. ${ }^{3}$

Surgical wound dehiscence (SWD) is defined as partial or total separation of previously approximated wound edges, due to a failure of proper wound healing up to and including 30 days postoperatively). ${ }^{4}$

Wound dehiscence according to the Centers for Disease Control and Prevention definition classifies dehiscence as surgical site infection regardless of whether the dehiscence is confirmed as microbial or of a non-microbial nature. ${ }^{5}$

It is divided into Superficial dehiscence involving the skin wound alone, with the rectus sheath remaining intact and Full thickness dehiscence where the rectus sheath fails to heal and bursts, with protrusion of abdominal content "burst abdomen".

Wound complication, even if not accompanied by an infection, is a significant cause of postoperative morbidity following cesarean 


\section{Biomedical and Case Reports Open Access Open Journal}

\section{Review}

delivery. In addition to the increased cost of care, there is the inconvenience of therapy, increased postoperative pain and convalescence, as well as difficulty with activities of daily living. ${ }^{?}$

It is logical to employ novel risk reducing approaches including negative pressure wound therapy at the time of surgery that may prevent wound complications and to ensure that there is a demonstrable benefit to their use for wound complication prophylaxis. ${ }^{8}$

Negative pressure wound therapy (NPWT), also known as a vacuum assisted closure (VAC), involves the controlled application of sub-atmospheric pressure to the local wound environment, using a sealed wound dressing connected to a vacuum to promote healing by primary intention by reducing the risk of hematoma / seroma due to improved lymphatic drainage and reduces the risk of wound dehiscence by decreasing the lateral and shear stress on sutures and decreasing bacterial load and wound fluids and by increasing blood flow, oxygenation, angiogenesis, and epithelialization. ${ }^{\text {? }}$

Recent NICE medical technologies guidance supports the case for adopting negative pressure wound dressings for closed surgical incisions. They are associated with fewer surgical site infections, seromas and wound dehiscence compared with standard wound dressings. ${ }^{10}$

On the contrary, RCOG review of clinical effectiveness and guidelines showed no relevant evidence regarding the clinical effectiveness of negative pressure wound dressings versus different surgical dressing types was identified. ${ }^{11}$

A significant reduction in wound dehiscence on the basis of pooled data from 16 studies showed a benefit of the NPWT system compared with standard care in closed surgical incisions was demonstrated by a meta-analysis by Strugala in $2017 .^{12}$

The 2020 Cochrane review of negative pressure wound therapy for surgical wounds healing by primary closure found fourteen studies which reported on dehiscence, the evidence suggested no clear difference between NPWT and standard dressing. ${ }^{13}$

Hence, it was prudent that we further study the effectiveness of Negative pressure wound therapy on closed surgical wounds..

\section{AIM / OBJECTIVES}

The objective of this study is to determine whether negative pressure wound therapy reduces the risk of post-cesarean wound dehiscence among obese women compared with standard surgical wound care.

\section{RESEARCH QUESTION}

Does negative pressure wound therapy reduce the risk of post-cesarean wound dehiscence among obese women compared with standard surgical wound care?

\section{Research Hypothesis}

In this study we hypothesize that negative pressure wound therapy reduces the risk of post-cesarean wound dehiscence among obese women compared with standard surgical wound care.

\section{METHODOLOGY}

Patients and Methods/ Subjects and Methods/ Material and Methods

\section{Type of Study}

Randomized controlled trial (RCT).

\section{Study Setting}

This study will be conducted at Ain Shams University Maternity Hospital.

\section{Study Period}

This study will be conducted in the period from July 2020 till the required number of study subjects is recruited and all eligible patients finish the work up planned in our study.

\section{Study Population}

This study will be conducted on women undergoing cesarean section at Ain Shams University Maternity Hospital.

\section{Inclusion Criteria}

Age

18 or older.

\section{BMI}

$30 \mathrm{~kg} / \mathrm{m} 2$ or greater (calculated by the weight in kilograms divided by the height in meters squared).

Undergoing Cesarean section through a Pfannenstiel incision.

\section{EXCLUSION CRITERIA}

Prolonged Rupture of membranes $>18$ hours as it is an independent risk factor for impaired wound healing .

\section{Intra Amniotic Infection}

Both bacteria and endotoxins can lead to the prolonged elevation of pro-inflammatory cytokines and elongate the inflammatory phase, increased level of matrix metalloproteases (MMPs), a decreased level of the naturally occurring protease inhibitors occurs. This shift in protease balance can cause growth factors that appears in chronic wounds to be rapidly degraded.

\section{Severe Anemia(impaired tissue oxygenation)}

Vasculopathies as in hypertension/pre-eclampsia, pre-gestational / gestational diabetes mellitus, smoking and substance abuse.(due to impaired tissue perfusion and oxygenation)

\section{Prolonged Steroids Therapy as in SLE, ITP}

Systemic steroids are well-known to inhibit wound repair via global anti-inflammatory effects and suppression of cellular wound responses, including fibroblast proliferation and collagen synthesis)

Intraoperative complications as bladder or bowel injury, placenta previa / accreta, difficult fetal extraction, ovarian cysts, pelvic abscess(which would lead to prolonged operative time and intra-opera- 


\section{Biomedical and Case Reports Open Access Open Journal}

\section{Review}

tive blood loss)

Intra-abdominal or subcutaneous drain as they are alternative drainage methods.

\section{SAMPLING METHOD}

\section{Convenient Sample}

Sample size: The study will include 260 patients divided into 2 groups of 130 patients each.

Sample size justification: Using PASS II program for sample size calculation and according to a previous study done by the expected rate of wound dehiscence in intervention group $=2.6 \%$ and in control group $=11.6 \%$, a sample size of 130 women /group can detect this difference with power $80 \%$ and $\alpha$-error 0.05 .

\section{Ethical Considerations}

The study will be performed after ethical committee approval, the study protocol will be explained to the patients, and written informed consent will be obtained from the patients

Study interventions: All patients participated in this study will undergo the following procedures:

\section{Full History Including}

Personal history: Name, age, ethnicity and special habits of medical importance (smoking or substance abuse).

Obstetric history: Gravidity, parity, last menstrual period, gestational age at time of delivery, previous cesarean delivery, prolonged rupture of membranes ( $>18$ hours), pre-operative fever and pre-operative diagnosis of intra-amniotic infection.

Medical history: Presence of chronic hypertension, pregnancy induced hypertension or pre-eclampsia, pre-gestational or gestational diabetes, chronic liver or kidney diseases, maternal auto-immune disease, haemoglobinopathy e.g. sickle cell anemia or long-term steroid use (more than 3 months).

Surgical history: History of SSI, previous abdominal or pelvic surgeries.

\section{Examination}

Measurement: Weight, height, body mass index (BMI) (calculated by the weight in kilograms divided by the height in meters squared), blood pressure and temperature.

\section{Laboratory Investigations}

$\mathrm{CBC}$

\section{PT, PTT, INR.}

Random blood sugar (RBS).

Liver function tests (LFT).

Kidney function tests (KFT).

Informed written consent will be obtained from female patients meeting the selection criteria after full explanation of the study procedure.

All patients will receive standard antibiotic prophylaxis as per ACOG protocol for women with $(\mathrm{BMI}>30 \mathrm{~kg} / \mathrm{m} 2)$ : a dose of $2 \mathrm{~g}$ cefazolin intravenous infusion at least 30 to 60 minutes prior to the skin incision.

Cesarean section will be done through a Pfannenstiel incision.

Closure of the incision by interrupted PROLENE Polypropylene Sutures (non-absorbable, mono-filament suture material).

Wound dressing will be applied in a sterile fashion.

The intervention group: placement of a sterile multilayer dressing (gauze and occlusive adhesive tape) over the closed incision. The dressing's tubing will then be attached to a compact, portable negative-pressure therapy unit (Yuwell 7E-A portable suction unit) that will deliver $-80 \mathrm{~mm} \mathrm{Hg}$ of continuous pressure to the dressing and will remove exudates into a disposable canister for 4 days.

The control group: use of traditional sterile wound dressing of gauze and tape for 4 days.

Post-operative data will be collected immediately post-operative and through follow up visits up to 30 days post-partum including:

Type dressing used (categories included gauze with tape or EPNDS).

Postoperative hemoglobin level.

Degree of post-operative pain assessed by Wong-Baker Faces pain rating scale.

Length of hospital stay (standard hospital stay will be 4 days unless complications occur).

Need for post-operative antibiotic.

Readmission due to surgical site infection.

Type of the surgical site wound complication.

In the case of SSI in either groups the patient will be managed as follows The patient will be re-admitted and will be subjected to:

Lab investigations: (Full blood count, liver functions, kidney functions, fasting and post prandial blood glucose levels, coagulation profile)

Wound discharge culture and sensitivity

In case of persistent fever or suspected endometritis and intra-abdominal communication the patient will be subjected to imagining by trans-vaginal ultrasound, abdominal $\mathrm{x}$-ray and pelvi-abdominal CT with contrast if needed.

Small hematomas and seromas will resorb without surgical interventions with frequent wound dressing.

Larger hematomas might need evacuation of the clot under sterile conditions, ligation or cauterization of bleeding vessels, and reclosure of the wound. 


\section{Biomedical and Case Reports Open Access Open Journal}

\section{Review}

Larger seromas will evacuated by needle aspiration. To prevent re-accumulation, compression dressings will be applied.

Superficial infection such as cellulitis will be treated with antibiotics. Options for oral antibiotics include dicloxacillin, cefadroxil, cephalexin, and clindamycin to cover beta-hemolytic streptococci and methicillin-sensitive Staphylococcus aureus (MSSA).

Wound separation will be managed by. Dressing changes several times daily will be performed, placing moistened gauze into the wound with covering by dry gauze. After the wound is clean they will be subject to delayed closure. Infected wounds will be left open to heal by secondary intention.

If the wound has purulent drainage, incision and drainage, sharp debridement until healthy tissue is identified and wound exploration to confirm the integrity of fascia will be done.

Fascial dehiscence is a surgical emergency and requires further wound exploration and closure.

Necrotizing fasciitis will be managed by surgical exploration and debridement of necrotic tissue in addition to broad-spectrum antibiotic therapy including agents effective against aerobes, including methicillinresistant Staphylococcus aureus, and anaerobes. Acceptable choices for antibiotics are vancomycin, linezolid, or daptomycin combined with one of the following options: (piperacillin-tazobactam, carbapenem, ceftriaxone plus metronidazole, fluoroquinolon plus metronidazole).

Endometritis will be treated by clindamycin $(900 \mathrm{mg}$ intravenously every $8 \mathrm{~h}$ ) plus gentamicin $(1.5 \mathrm{mg} / \mathrm{kg}$ [ideal body weight] every $8 \mathrm{~h}$ ). Ampicillin may be added to the regimen for better coverage of enterococcus.

\section{Outcome Measures}

Primary outcome: The incidence of wound dehiscence defined as the separation of the incision line prior to complete healing resulting in an open wound on the day of removal of the stitches( $\left(7^{\text {th }}\right.$ day).

\section{Secondary outcomes:}

The incidence of surgical site infections (as defined by the CDC).

SSIs is divided into incisional SSI and organ/space SSI up to 30 days post-operative. with at least one of the following:

Purulent drainage, with or without laboratory confirmation, from the superficial incision.

Organisms isolated from an aseptically obtained culture of fluid or tissue from the superficial incision.

At least one of the following signs or symptoms of infection: pain or tenderness, localized swelling, redness, or heat and superficial incision is deliberately opened by surgeon, unless incision is culturenegative

Diagnosis of superficial incisional surgical site infection (SSI) by surgeon or attending physician.
Incisional SSI is further divided into superficial and deep incisional SSI. Superficial Incisional Surgical Site Infection involves skin or subcutaneous tissue cellulitis, seroma and hematoma. Deep Incisional Surgical Site Infection involves deep soft tissues such as fascia or muscle within incision. Organ/Space Surgical Site Infection involves any part of the anatomy other than the incision.

Degree of post-operative pain.

Length of hospital stay.

Need for readmission.

Further need for postoperative antibiotics.

Incidence of peri-incisionl skin blistering.

Patient mobility.

\section{STATISTICAL ANALYSIS}

The collected data will be coded, tabulated, and statistically analyzed using IBM SPSS statistics (Statistical Package for Social Sciences) software version 22.0, IBM Corp., Chicago, USA, 2013. Descriptive statistics will be done for quantitative data as minimum\& maximum of the range as well as mean \pm SD (standard deviation) for quantitative normally distributed data, while it will be done for qualitative data as number and percentage. Inferential analyses will be done for quantitative variables using Shapiro-Wilk test for normality testing, independent t-test in cases of two independent groups with normally distributed data. In qualitative data, inferential analyses for independent variables will be done using Chi square test for differences in between. ROC curve will be used to evaluate the performance of different tests. The level of significance will be taken at $P$ value $<0.050$ is significant, otherwise is non-significant.

\section{CONFLICTS OF INTEREST}

None.

\section{REFERENCES}

1. Smid MC, Dotters-Katz SK, Grace M, Wright et al. Prophylactic negative pressure wound therapy for obese women after cesarean delivery: A systematic review and meta-analysis. Obstetrics \& Gynecology. 2017; 130(5): 969-978. doi: 10.1097/AOG.0000000000002259

2. Mark KS, Alger L, Terplan M. Incisional negative pressure therapy to prevent wound complications following cesarean section in morbidly obese women: A pilot study. Surgical innovation. 2014; 21(4): 345-349. doi: 10.1177/1553350613503736

3. Pai V, Aboosalih S, Balaji D, et al. The role of negative suction drains in exploratory laparotomy for peritonitis. International Journal of Scientific Research. 2020; 8(11): 19-21. doi: 10.36106/IJSR

4. Sandy-Hodgetts K, Carville, K, Leslie GD. Surgical wound dehiscence: a conceptual framework for patient assessment. Journal of wound care. $2018 ; 27(3), 119-126$.

5. Gommesen D, Nohr EA, Drue HC, et al. Obstetric perineal tears: Risk factors, wound infection and dehiscence: A prospective cohort 


\section{Biomedical and Case Reports Open Access Open Journal}

\section{Review}

study. Archives of gynecology and obstetrics. 2019; 300(1): 67-77. doi: 10.1007/s00404-019-05165-1

6. Clark TJ. Wound Dehiscence. Gynecologic and Obstetric Surgery: Challenges and Management Options. 2016; 147.

7. Orth, TA, Gerkovich MM, Heitmann E, et al. Cesarean delivery with external negative pressure dressing system: A retrospective cohort study. The Surgery Journal. 2016; 2(03): e59-e65. doi: 10.1055/s-0036-1585470

8. Wells C I, Ratnayake CB, Perrin J, et al. Prophylactic Negative Pressure Wound Therapy in closed abdominal incisions: A meta-analysis of randomized controlled trials. World Journal of Surgery. 2019; 43(11): 2779-2788. doi: 10.1007/s00268-019-05116-6

9. Scalise A, Tartaglione C, Bolletta E, et al. The enhanced healing of a high-risk, clean, sutured surgical incision by prophylactic negative pressure wound therapy as delivered by Prevena ${ }^{\text {Txt }}$ Customizable $^{\text {trut: }}$ cosmetic and therapeutic results. International wound journal. 2015; 12(2): 218-
223. doi: 10.1111 iwj. 12370

10. Skervin A, Levy B. Management of common surgical complications. Surgery (Oxford). 2020; 38(3): 128-132.

11. Gray C, Farrah K. Post-operative Procedures for Caesarean Sections: A Review of Clinical Effectiveness and Guidelines. Canadian Agency for Drugs and Technologies in Health Rapid Response Reports.

12. Strugala V, Martin R. Meta-analysis of comparative trials evaluating a prophylactic single-use negative pressure wound therapy system for the prevention of surgical site complications. Surgical infections. 2017; 18(7): 810-819. doi: 10.1089/sur.2017.156

13. Norman G, Goh EL, Dumville JC, et al. Negative pressure wound therapy for surgical wounds healing by primary closure. Cochrane Database of Systematic Reviews. 2020; (5). doi: 10.1002/14651858.CD009261. pub4 